\title{
Enhancing The Photocatalytic Conversion Of Pt(IV) Substrates By Flavoprotein Engineering
}

\author{
Juan Gurruchaga-Pereda, a,b Virginia Martínez-Martínez, ${ }^{\mathrm{c}}$ Elena Formoso, a,d Oksana \\ Azpitarte, ${ }^{\text {a,e }}$ Elixabete Rezabal, ${ }^{\text {a,e }}$ Xabier Lopez, ${ }^{\text {a,e }}$ Aitziber L. Cortajarena, ${ }^{* b, f}{ }^{\text {Luca Salassa*a,e,f }}$ \\ a Donostia International Physics Center, Paseo Manuel de Lardizabal 4, Donostia, 20018, \\ Spain \\ ${ }^{b}$ Center for Cooperative Research in Biomaterials (CIC biomaGUNE), Basque Research and \\ Technology Alliance (BRTA), Paseo de Miramón 194, Donostia San Sebastián, 20014, Spain \\ c Departamento de Química Física, Universidad del País Vasco, UPV/EHU, Apartado 644, \\ Bilbao, 48080, Spain \\ ' Kimika Fisikoa, Farmazia Fakultatea, Euskal Herriko Unibertsitatea UPV/EHU, Paseo de la \\ Universidad 7, Vitoria-Gasteiz, 01006, Spain \\ e Polimero eta Material Aurreratuak: Fisika, Kimika eta Teknologia, Kimika Fakultatea, Euskal \\ Herriko Unibertsitatea UPV/EHU, Paseo Manuel de Lardizabal 3, Donostia, 20018, Spain \\ ${ }^{f}$ Ikerbasque, Basque Foundation for Science, Bilbao, 48011, Spain
}

\begin{abstract}
Our recent work demonstrates that certain flavoproteins can catalyze the redox activation of $\mathrm{Pt}(\mathrm{IV})$ prodrug complexes under light irradiation. Herein, we used site directed mutagenesis on the mini Singlet Oxygen Generator (mSOG) to modulate the photocatalytic activity of this flavoprotein towards two model Pt(IV) substrates. Among the prepared mutants, Q103V mSOG displayed enhanced catalytic efficiency as a result of its longer triplet excited state lifetime. This study shows, for the first time, that protein engineering can improve the catalytic capacity of a protein towards metal-containing substrates.
\end{abstract}

Flavoproteins and flavoenzymes are capable of photocatalyzing the reduction of metal complexes in the presence of electron donors. ${ }^{1}$ Recently, we demonstrated that flavoproteins such as NOX (NADH oxidase) enzyme and mSOG (mini Singlet Oxygen Generator) converted Pt(IV) anticancer agents into their biological active Pt(II) counterparts (e.g. cisplatin) with remarkable turnover numbers (TONs) and frequencies (TOFs), including in biological environments. ${ }^{2-4}$ Key steps in the catalytic mechanism of these reactions are the light-induced formation of the flavin triplet-excited state $\left({ }^{3} \mathrm{FL}^{*}\right)$ and its subsequent reductive quenching by electron donors, which affords the flavin hydroquinone form (e.g. $\mathrm{HFL}^{-}$) capable of reducing Pt(IV) substrates (Figure 1a).

Besides providing one of the rare examples of catalytic reactions that use metal complexes as substrates, ${ }^{5,6}$ this unconventional chemistry has interest in the design of new strategies for the (photo)activation of metal-based prodrugs and their application in photochemotherapy. ${ }^{4,7}$

Considering the spectacular results obtained by protein engineering in catalysis, ${ }^{8,9}$ we wondered how modification of flavoprotein structures could affect their capacity to catalytically transform Pt(IV) substrates and whether or not site-directed mutagenesis could 
enhance their activation efficiency upon light irradiation. For this reason, we selected the FMN-containing mSOG flavoprotein and investigated the catalytic performance of its Q103V, Q50E and Q50W mutants with respect to the wild type (WT). mSOG is a suitable model for this task since mutants of this protein and their photophysics have been thoroughly studied for use in correlative light and electron microscopy (CLEM) and for ${ }^{1} \mathrm{O}_{2}$ sensitization purposes. ${ }^{10-15}$

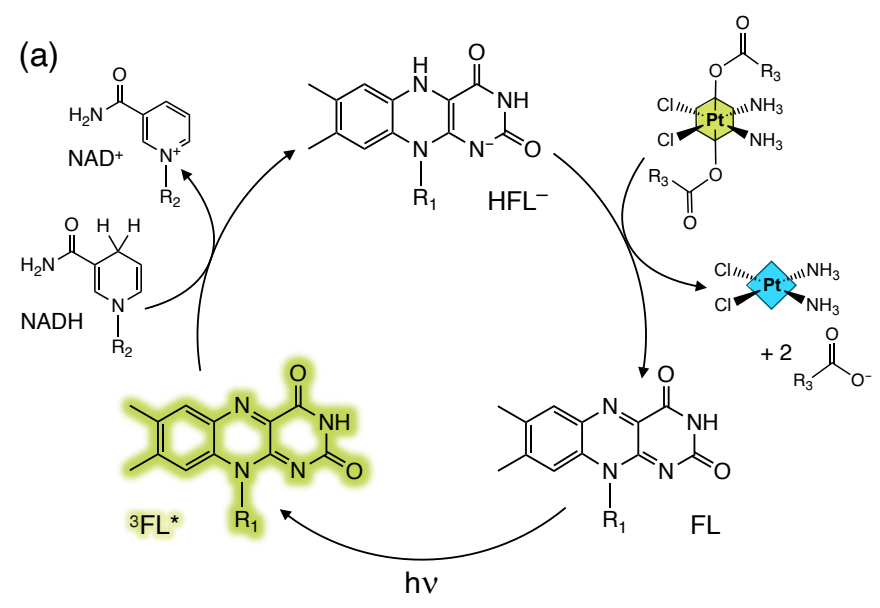

(b)

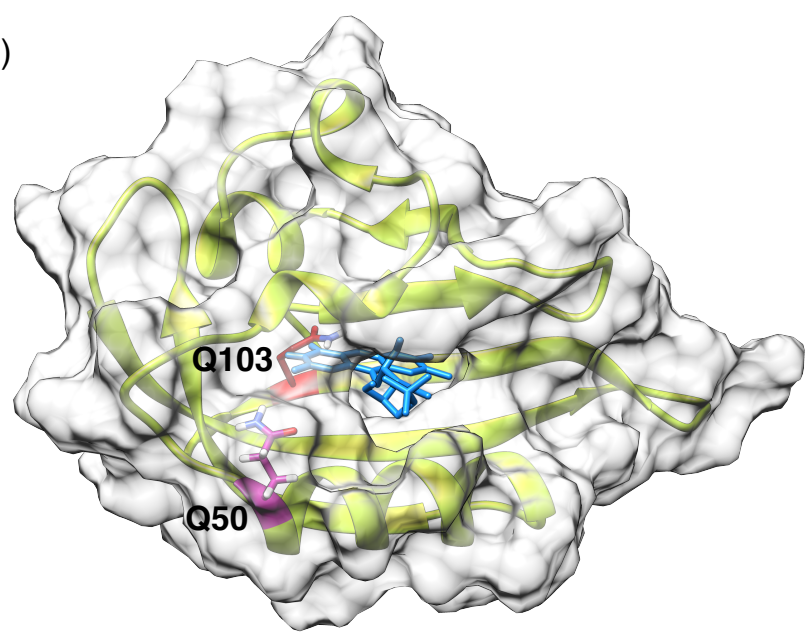

Figure 1. (a) General photocatalytic mechanism for the Pt(IV) prodrug activation by flavins; $\mathrm{R}_{1}=$ e.g. ribityl, ribityl phosphate, $\mathrm{R}_{2}$ = adenosine diphosphate ribose and $\mathrm{R}_{3}=\mathrm{CH}_{2} \mathrm{CH}_{2} \mathrm{COOH}$ (1) or $\mathrm{CH}_{3}$ (2). (b) mSOG's molecular model based on the structure of iLOV protein (PBD ID: 6GPU). The backbone of mSOG is shown as a yellow ribbon, FMN as blue sticks, and the amino acids in the mutation positions in violet (Q50) and red (Q103).

The photophysics of Q103V mutant and its Q103L (SOPP, singlet oxygen photosensitizing protein) analogue were previously investigated by several groups. ${ }^{12,14,15}$ The glutamine residue in the 103 position has been suggested to form a hydrogen bond with FMN (Figure 1b), likely enhancing quenching of ${ }^{3} \mathrm{FMN}^{*}$ and formation of $\mathrm{O}_{2} \cdot{ }^{\cdot-} \cdot{ }^{15}$ Substitution of the $Q$ residue for an amino acid with an hydrophobic side chain such as valine or leucine proved to increase significantly the ${ }^{3} \mathrm{FMN}^{*}$ lifetime and the overall capacity of mSOG to sensitize ${ }^{1} \mathrm{O}_{2} \cdot{ }^{12,14,15} \mathrm{Q} 50 \mathrm{E}$ and $\mathrm{Q} 50 \mathrm{~W}$ are instead novel mutants that we generated based on the proximity of the Q50 position to the FMN-containing pocket (Figure 1b), with the aim of evaluating the effects of a negatively charged (glutamic acid, E, $\mathrm{pK}_{\mathrm{a}} 4.5$ ) and a bulky aromatic amino acid (tryptophan, $W$ ) on the catalysis. Furthermore, $W$ is known to be optically active and an excited-state quencher for flavins. ${ }^{16,17}$ 
In order to test the capacity of the different mutants to catalyze the conversion of Pt(IV) complexes, we employed the cisplatin prodrugs cis, cis,trans$\left[\mathrm{Pt}\left(\mathrm{NH}_{3}\right)_{2}(\mathrm{Cl})_{2}\left(\mathrm{O}_{2} \mathrm{CCH}_{2} \mathrm{CH}_{2} \mathrm{CO}_{2} \mathrm{H}\right)_{2}\right](1)$ and cis, cis, trans-[Pt $\left(\mathrm{NH}_{3}\right)_{2}\left(\mathrm{Cl}_{2}\left(\mathrm{O}_{2} \mathrm{CCH}_{3}\right)_{2}\right](2)$, together with $\mathrm{NADH}$ as electron donor (Figure $1 \mathrm{a}$ ).

To the best of our knowledge this is the first attempt to engineer a protein for enhancing a catalytic process involving the transformation of metal-containing compounds. These studies may have key implications in biology as well as in the development of new technologies for medicine.

Firstly, we characterized the photophysical and photochemical properties of the four mSOG catalysts employing different optical methods and experimental conditions (Figure S1-S8). The four proteins displayed absorption and emission profiles typical of the FMN chromophore, with fluorescence lifetimes falling in the 4-5 ns range (Figure S1). The Q50W mutant had the shortest fluorescence lifetime (4 ns) indicating that the $W$ residue favors deactivation of the ${ }^{1} \mathrm{FMN}$ excited state, which confirmed the expected coupling between $\mathrm{W}$ and FMN from the design hypothesis. Transient absorption spectra for the mSOG mutants and the WT showed the characteristic features of ${ }^{3} \mathrm{FMN}$, that is a negative band for groundstate bleaching at $420-480 \mathrm{~nm}$ and a positive band at 550-750 nm, corresponding to the absorption of the T1 state (Figure 2). ${ }^{2,16}$ We determined the ${ }^{3} \mathrm{FMN}$ lifetime in aerated solutions by monitoring the mono-exponential decay of the triplet-triplet absorption at 720 nm (Figure 2 and Figure S2). Compared to mSOG WT (35 $\mu \mathrm{s})$, Q103V and Q50W mutants showed triplet lifetimes $\left(\tau^{\top}\right)$ that were significantly longer and shorter respectively (102 vs $1.8 \mu \mathrm{s})$. The dramatic $\mathrm{\tau}^{\top}$ reduction observed for Q50W can be attributed to electron transfer processes involving the tryptophan and ${ }^{3} \mathrm{FMN}$ moieties, including the formation of radical pairs. ${ }^{16,17}$ Conversely, Q50E resembled the WT displaying a ${ }^{3} \mathrm{FMN}$ decay of $31 \mu \mathrm{s}$. The lifetime values obtained in this work for WT and Q103V are consistent with data previously reported. ${ }^{11}$
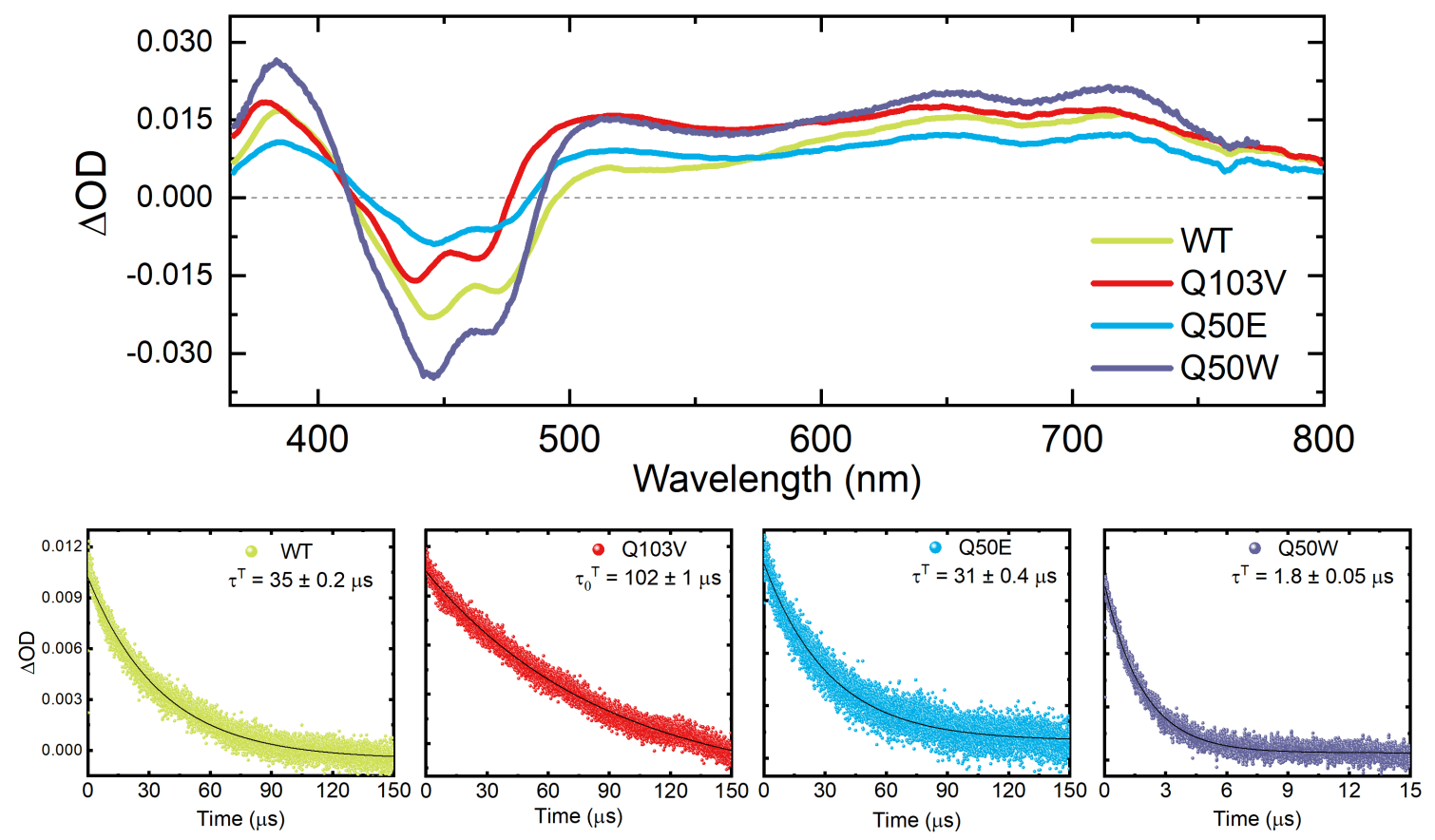

Figure 2. Transient absorption spectra $\left(\lambda_{\text {exc }}=445 \mathrm{~nm}\right)$ and decays $\left(\tau^{\top}\right)$ of the triplet-triplet absorption at $720 \mathrm{~nm}$ for mSOG WT and its Q103V, Q50E and Q50W mutants. All measurements were performed in $\mathrm{PB}$ solutions ( $20 \mathrm{mM}, \mathrm{pH}$ 7, air-saturated) of the $\mathrm{mSOG}$ proteins. 
Photostability is pivotal in the performance of a photocatalyst, hence we investigated the behavior of the four $\mathrm{mSOGs}$ under light irradiation $\left(460 \mathrm{~nm}, 6 \mathrm{~mW} \cdot \mathrm{cm}^{-2}\right.$ ) and different experimental conditions. In oxygenated solutions, all samples showed a decrease in the absorbance band at $450 \mathrm{~nm}$, characteristic of FMN degradation (Figure S3 and S4). ${ }^{14,15,18,19}$ The WT and mutants displayed rather similar photodecomposition rates at short irradiation times ( $<10 \mathrm{~min}$ ), that is in the timeframe of the catalysis experiments (vide infra). When exposed to blue light for longer time periods, a band at $600 \mathrm{~nm}$ was observed in the spectra of the WT and Q50W, corresponding to the formation of an FMN radical (semiquinone). This species is observed particularly in oxygen-free conditions where the ${ }^{3} \mathrm{FMN} *$ can be efficiently deactivated by electron transfer processes involving the mSOG amino acidic scaffold. ${ }^{15}$ Furthermore, after prolonged irradiations (ca. > $20 \mathrm{~min}$ ), Q103V underwent photodecomposition in a more pronounced manner compared to the other mSOG forms. Such result is consistent with the increased capacity of this mutant to produce ${ }^{1} \mathrm{O}_{2}$ and other ROS (especially $\mathrm{O}_{2}{ }^{-}$), as determined by indirect methods validated previously for $\mathrm{mSOG}$ (Figure S5 and S6). ${ }^{13,18}$

The formation of the FMN radical was more pronounced for all mSOG variants in the absence of $\mathrm{O}_{2}$ (Figure $\mathrm{S7}$ ), which acts as quencher of the $\mathrm{FMN}$ radical anion $\left(\mathrm{FMN}^{{ }^{-}}\right.$) to give $\mathrm{O}_{2}{ }^{--}$via a second electron transfer. Importantly, the $\mathrm{FMN}^{*-}$ rapidly disappeared when sample were reexposed to oxygen-containing ambient atmosphere. Concomitantly, the FMN-associated band at 400-500 $\mathrm{nm}$ recovered almost completely, although this could be in part ascribed to the formation of lumichrome. ${ }^{15}$ Among the mSOG proteins, Q103V and Q50W showed the lowest formation of $\mathrm{FMN}^{-}$. In the case of the former, previous work demonstrated that mutation of the Q103 position led to reducing the rate of electron transfer from the protein to $\mathrm{FMN}^{14,18}$ For the latter instead, the generation of $\mathrm{FMN}^{\bullet-} / \mathrm{Trp}^{{ }^{*+}}$ radical pairs and back electron transfer processes may explain the low intensity of the 600-nm band, as well as the shorter ${ }^{3} \mathrm{FMN}^{*}$ lifetime observed by transient absorption. ${ }^{17}$ Notably, circular dichroism spectra of light-irradiated mSOG variants showed that formation of the FMN radical did not cause major changes in the protein structure (Figure S8).

Next, we evaluated how the photophysical and photochemical properties of the different mSOG proteins impacted the catalytic transformation of complexes 1 and 2 . Catalysis experiments were run using PB solutions containing $25 \mu \mathrm{M}$ mSOG (5\% loading), $500 \mu \mathrm{M}$ $\mathrm{Pt}(\mathrm{IV})$ substrate and $1 \mathrm{mM} \mathrm{NADH}$. The progression of the reactions in the dark and under light irradiation was monitored by ${ }^{1} \mathrm{H}$ NMR as explained in the Supporting Information.

Initial control experiments confirmed that the substrate conversion was rather ineffective in the dark (Figure S9 and S10) or without $\mathrm{mSOG}^{2}{ }^{2}$ We detected no activity in the first hours and only $20 \%$ of 1 and $60-80 \%$ of 2 were transformed by mSOG WT and mutants after $48 \mathrm{~h}$ in the absence of light. On the contrary, under light irradiation, the reactions were considerably faster for both 1 and 2 (Figure 3, Figure S11 and S12). As summarized in Table 1, all photocatalysts practically achieved full conversion of the substrates within 5 min (1.8 $\mathrm{J} \cdot \mathrm{cm}^{-2}$ ), corresponding to turnover numbers (TON) of 20. In addition, experiments with reduced photocatalyst loadings (0.1\%) afforded TON $N_{\max }$ values comprised between 610 and 738 within 30 min of light irradiation (Figure S13). 


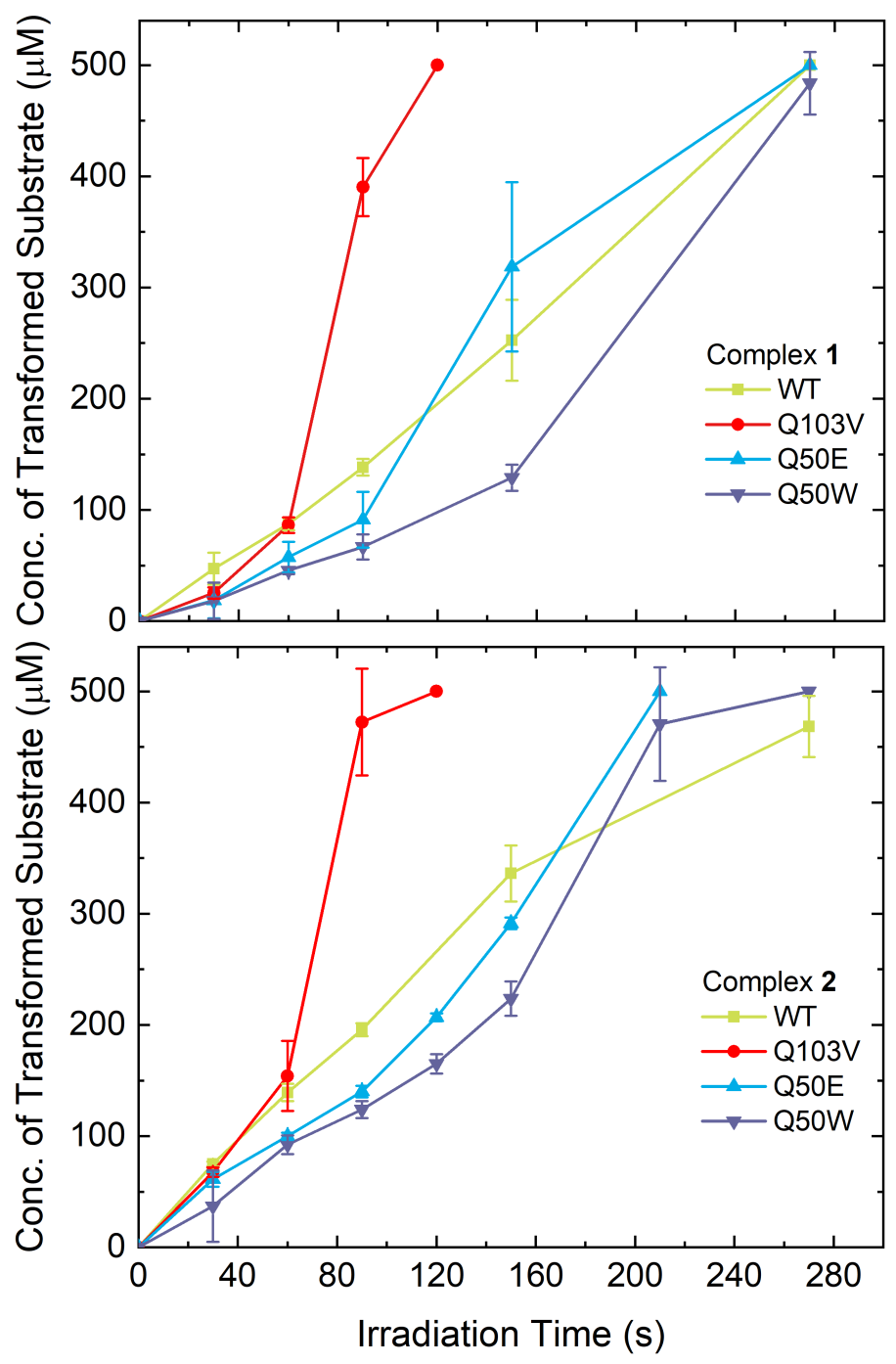

Figure 3. Kinetics profiles for the photocatalytic conversion of 1 and 2 by mSOG variants. Reactions were monitored by NMR and performed in PB solutions (18 mM PB, pH 7.0, 10\% $\mathrm{D}_{2} \mathrm{O}$ ) containing 25 $\mu \mathrm{M}$ mSOG catalysts, $500 \mu \mathrm{M} 1$ or 2 and $1 \mathrm{mM}$ NADH.

Anyhow, as expected from the photophysical studies, Q103V displayed the highest catalytic activity among all proteins. Already after $90 \mathrm{~s}$ of light irradiation, this mutant converted approximately more than $80 \%$ of $\mathrm{Pt}(\mathrm{IV})$ substrate. For instance, in the case of 1 , this corresponded to 2.8- and 5.8-fold increase in the turnover frequency (TOF) compared to WT and Q50W, respectively. Results also showed that Q50E behaved rather similarly to WT in terms of catalytic efficiency (Table 1 ).

The long ${ }^{3} \mathrm{FMN} *$ lifetime of Q103V is crucial for its enhanced catalytic activity since it favors $\mathrm{NADH}$-promoted reductive quenching, ${ }^{2}$ which ultimately results in higher concentrations of the flavin hydroquinone $\mathrm{HFL}^{-}$, that is the active catalyst that converts $\mathrm{Pt}(\mathrm{IV})$ prodrugs in cisplatin. It is also worth highlighting that the presence of Pt(IV) substrate and NADH did not induce significant changes in the capacity of the proteins to undergo electron transfer reactions and form the radical anion species (Figure S14 and Figure S15). Such finding suggests that the protein quenching channel of ${ }^{3} \mathrm{FMN}^{*}$ is still active under the catalysis conditions and competes with the substrate conversion. 
Table 1. Turnover Frequencies (TOFs, $\mathrm{min}^{-1}$ ), Turnover Numbers (TONs), and conversion (Conv.) Percentages for mSOG mutants catalyzed Photoactivation of 1 and 2 in the presence of NADH.

\begin{tabular}{|c|c|c|c|c|}
\hline Complex & TOF $\left(\min ^{-1}\right)$ & TON & Conv. (\%) & TON $_{\max }$ \\
\hline \multicolumn{5}{|c|}{ WT } \\
\hline 1 & $3.7 \pm 0.2$ & 20 & 100 & 610 \\
\hline 2 & $5.2 \pm 0.2$ & 19 & 94 & - \\
\hline \multicolumn{5}{|c|}{ Q103V } \\
\hline 1 & $10.4 \pm 0.7^{*}$ & 20 & 100 & 673 \\
\hline 2 & $12.6 \pm 1.3^{*}$ & 20 & 100 & - \\
\hline \multicolumn{5}{|c|}{ Q50E } \\
\hline 1 & $2.4 \pm 0.7$ & 20 & 100 & 738 \\
\hline 2 & $3.7 \pm 0.1$ & 20 & 100 & - \\
\hline \multicolumn{5}{|c|}{ Q50W } \\
\hline 1 & $1.8 \pm 0.3$ & 20 & 100 & 734 \\
\hline 2 & $3.3 \pm 0.2$ & 20 & 100 & - \\
\hline
\end{tabular}

We gained further insights by performing dynamic molecular simulations of the WT, Q103V and Q50W bearing the FMN in its oxidized form. All $\mathrm{mSOG}$ variants showed rather similar structure and FMN-protein interaction pattern, with the isoalloxazine ring and ribytil chain of the FMN buried in the protein binding pocket, leaving the phosphate group accessible through an entrance channel. Overall, during the simulation, the residue in the 50 position ( $Q$ or W) alternated between two conformations (Figure S16 and S17), being oriented either towards the interior of the channel ("closed") or outwards ("open"). None of these two conformations appeared able to hinder the passage through the channel in the case of Q103V or WT, hence suggesting that the higher catalytic activity of such mutant compared to WT could be ascribed exclusively to the ${ }^{3} \mathrm{FMN}^{*}$ excited state properties and the lack of quenching from the residue in position 103.

Conversely, few differences were observed in the case of Q50W. Although the entrance channel of this mutant remained open during most of the simulation (contrary to Q103V and WT), its "closed" conformation was more hindered, due to the bulky nature of the W residue. More importantly, we observed that the non-polar nature of the $\mathrm{W}$ side chain reduced the diffusion of charged particles such as $\mathrm{Na}^{+}$ions through the channel to the FMN phosphate group, even in the open channel conformation (Figure S18). The finding indicates that this residue establishes attractive interactions enabling the access to the phosphate group in FMN. Similar behavior should be expected for other charged or polar molecules, i.e. Pt(IV) substrates or NADH, inferring that the lowest catalytic activity of Q50W might not only be linked to its excited state features but also to a less efficient entrance channel.

In conclusion, our proof-of-concept study demonstrates for the first time that site directed mutagenesis can be employed to alter and enhance the catalytic activity of flavoproteins in artificial reactions involving metal complexes as substrates. Such a result was principally achieved by tuning the mSOG photophysics properties via protein engineering. Moreover, we envision that far greater improvements could be accomplished introducing structural modifications in flavoproteins that are capable of influencing substrate binding and product release steps of catalytic reactions. Currently, we seek to reach such goals by tailoring flavoenzymes that have higher structural and functional complexity than mSOG. 


\section{AUTHOR INFORMATION \\ Corresponding Author}

E-mail*: alcortajarena@cicbiomagune.es

E-mail*: Isalassa@dipc.org

ORCID

Virginia Martínez-Martínez: 0000-0001-7551-3714

Elena Formoso: 0000-0002-7106-3717

Oksana Azpitarte: 0000-0003-0874-6424

Elixabete Rezabal: 0000-0003-0397-6140

Xabier Lopez: 0000-0002-2711-3588

Aitziber L. Cortajarena: 0000-0002-5331-114X

Luca Salassa: 0000-0002-2112-9095

\section{Notes}

The authors declare no competing financial interest.

\section{SUPPORTING INFORMATION}

Experimental details, stability and catalysis experiments, time-resolved spectroscopy and computational data.

\section{ACKNOWLEDGMENTS}

We acknowledge the Spanish State Research Agency for the grants PID2019-109111RB-I00, PGC2018-097529-B-100, PID2019-111649RB-I00, ERACoBioTech HOMBIOCAT-PCI2018092984, MAT2017-83856-C3-3-P and the Spanish Multi-MetDrugs network (RED2018102471-T) for fruitful discussion. We also thank Eusko Jaurlaritza (grants IT1254-19 and IT912-16) and the technical and human support provided by the IZO-SGI SGIker of UPV/EHU. A.L.C. received funding from the European Research Council (ERC-CoG-648071-ProNANO). This work was performed under the Severo Ochoa Centres of Excellence and Maria de Maeztu Units of Excellence Programs of the Spanish State Research Agency - Grant No. CEX2018-000867-S (DIPC) and MDM-2017-0720 (CIC biomaGUNE). 


\section{References}

(1) Alonso-de Castro, S.; Cortajarena, A. L.; López-Gallego, F.; Salassa, L. Bioorthogonal Catalytic Activation of Platinum and Ruthenium Anticancer Complexes by FAD and Flavoproteins. Angew. Chem. Int. Ed. 2018, 57 (12), 3143-3147. https://doi.org/10.1002/anie.201800288.

(2) Gurruchaga-Pereda, J.; Martínez-Martínez, V.; Rezabal, E.; Lopez, X.; Garino, C.; Mancin, F.; Cortajarena, A. L.; Salassa, L. Flavin Bioorthogonal Photocatalysis Toward Platinum Substrates. ACS Catal. 2020, 10 (1), 187-196. https://doi.org/10.1021/acscatal.9b02863.

(3) Alonso-de Castro, S.; Ruggiero, E.; Ruiz-de-Angulo, A.; Rezabal, E.; Mareque-Rivas, J. C.; Lopez, X.; López-Gallego, F.; Salassa, L. Riboflavin as a Bioorthogonal Photocatalyst for the Activation of a Pt IV Prodrug. Chem. Sci. 2017, 8 (6), 4619-4625. https://doi.org/10.1039/C7SC01109A.

(4) Alonso-de Castro, S.; Terenzi, A.; Hager, S.; Englinger, B.; Faraone, A.; Martínez, J. C.; Galanski, M.; Keppler, B. K.; Berger, W.; Salassa, L. Biological Activity of PtIV Prodrugs Triggered by Riboflavin-Mediated Bioorthogonal Photocatalysis. Sci. Rep. 2018, 8 (1), 17198. https://doi.org/10.1038/s41598-018-35655-2.

(5) Ruffoni, A.; Juliá, F.; Svejstrup, T. D.; McMillan, A. J.; Douglas, J. J.; Leonori, D. Practical and Regioselective Amination of Arenes Using Alkyl Amines. Nat. Chem. 2019, 11 (5), 426-433. https://doi.org/10.1038/s41557-019-0254-5.

(6) Gong, L.; Lin, Z.; Harms, K.; Meggers, E. Isomerization-Induced Asymmetric Coordination Chemistry: From Auxiliary Control to Asymmetric Catalysis. Angew. Chem. Int. Ed. 2010, 49 (43), 7955-7957. https://doi.org/10.1002/anie.201003139.

(7) Mazzei, L. F.; Martínez, Á.; Trevisan, L.; Rosa-Gastaldo, D.; Cortajarena, A. L.; Mancin, F.; Salassa, L. Toward Supramolecular Nanozymes for the Photocatalytic Activation of Pt(IV) Anticancer Prodrugs. Chem. Commun. 2020, 56 (72), 10461-10464. https://doi.org/10.1039/D0CC03450A.

(8) Chen, K.; Arnold, F. H. Engineering New Catalytic Activities in Enzymes. Nat. Catal. 2020, 3 (3), 203-213. https://doi.org/10.1038/s41929-019-0385-5.

(9) Arnold, F. H. Innovation by Evolution: Bringing New Chemistry to Life (Nobel Lecture). Angew. Chem. Int. Ed. 2019, 58 (41), 14420-14426. https://doi.org/10.1002/anie.201907729.

(10) Shu, X.; Lev-Ram, V.; Deerinck, T. J.; Qi, Y.; Ramko, E. B.; Davidson, M. W.; Jin, Y.; Ellisman, M. H.; Tsien, R. Y. A Genetically Encoded Tag for Correlated Light and Electron Microscopy of Intact Cells, Tissues, and Organisms. PLoS Biol. 2011, 9 (4), e1001041. https://doi.org/10.1371/journal.pbio.1001041.

(11) Westberg, M.; Bregnhøj, M.; Etzerodt, M.; Ogilby, P. R. Rational Design of an Efficient, Genetically Encodable, Protein-Encased Singlet Oxygen Photosensitizer. J. Phys. Chem. B 2017, 121 (12), 2561-2574. https://doi.org/10.1021/ja511940j.

(12) Westberg, M.; Bregnhøj, M.; Etzerodt, M.; Ogilby, P. R. Temperature Sensitive Singlet Oxygen Photosensitization by LOV-Derived Fluorescent Flavoproteins. J. Phys. Chem. B 2017, 121 (12), 2561-2574. https://doi.org/10.1021/acs.jpcb.7b00561.

(13) Ruiz-González, R.; Cortajarena, A. L.; Mejias, S. H.; Agut, M.; Nonell, S.; Flors, C. Singlet Oxygen Generation by the Genetically Encoded Tag MiniSOG. J. Am. Chem. Soc. 2013, 135 (26), 9564-9567. https://doi.org/10.1021/ja4020524.

(14) Rodríguez-Pulido, A.; Cortajarena, A. L.; Torra, J.; Ruiz-González, R.; Nonell, S.; Flors, C. Assessing the Potential of Photosensitizing Flavoproteins as Tags for Correlative 
Microscopy. Chem. Commun. 2016, 52 (54), 8405-8408. https://doi.org/10.1039/C6CC03119F.

(15) Torra, J.; Lafaye, C.; Signor, L.; Aumonier, S.; Flors, C.; Shu, X.; Nonell, S.; Gotthard, G.; Royant, A. Tailing MiniSOG: Structural Bases of the Complex Photophysics of a FlavinBinding Singlet Oxygen Photosensitizing Protein. Sci. Rep. 2019, 9 (1), 2428. https://doi.org/10.1038/s41598-019-38955-3.

(16) Cardoso, D. R.; Franco, D. W.; Olsen, K.; Andersen, M. L.; Skibsted, L. H. Reactivity of Bovine Whey Proteins, Peptides, and Amino Acids toward Triplet Riboflavin as Studied by Laser Flash Photolysis. J. Agric. Food Chem. 2004, 52 (21), 6602-6606. https://doi.org/10.1021/jf0401165.

(17) Bialas, C.; Barnard, D. T.; Auman, D. B.; McBride, R. A.; Jarocha, L. E.; Hore, P. J.; Dutton, P. L.; Stanley, R. J.; Moser, C. C. Ultrafast Flavin/Tryptophan Radical Pair Kinetics in a Magnetically Sensitive Artificial Protein. Phys. Chem. Chem. Phys. 2019, 21 (25), 13453-13461. https://doi.org/10.1039/C9CP01916B.

(18) Westberg, M.; Holmegaard, L.; Pimenta, F. M.; Etzerodt, M.; Ogilby, P. R. Rational Design of an Efficient, Genetically Encodable, Protein-Encased Singlet Oxygen Photosensitizer. J. Am. Chem. Soc. 2015, 137 (4), 1632-1642. https://doi.org/10.1021/ja511940j.

(19) Pimenta, F. M.; Jensen, R. L.; Breitenbach, T.; Etzerodt, M.; Ogilby, P. R. OxygenDependent Photochemistry and Photophysics of "MiniSOG," a Protein-Encased Flavin. Photochem. Photobiol. 2013, 89 (5), 1116-1126. https://doi.org/10.1111/php.12111. 